\title{
CROSSED TESTICULAR ECTOPIA-A RARE ANAMOLY
}

\author{
Noopur Priya ${ }^{1}$, Luv Luthra ${ }^{2}$, Sweta Agrawal ${ }^{3}$ \\ ${ }^{1}$ Resident, Department of General Surgery, Jawaharlal Nehru Medical College, Wardha, MAH, India \\ ${ }^{2}$ Senior resident, Department of General Surgery, Jawaharlal Nehru medical college, Sawangi, Meghe, Wardha, MAH, India \\ ${ }^{3}$ Assistant Professor, Department of Pathology, Jawaharlal Nehru medical college, Sawangi, Meghe, Wardha, MAH, India \\ Conflict of Interest - NIL, Received - 01/08/2015, Accepted - 07/09/2015, Published 10/09/2015
}

\begin{abstract}
Crossed Testicular Ectopia (CTE)/Transverse Testicular Ectopia (TTE) is a rare but well-known congenital anomaly, in which both gonads migrate toward the same hemiscrotum. It is usually associated with other abnormalities such as persistent Mullerian duct syndrome, True Hermaphroditism, Inguinal Hernia, Hypospadias, Pseudohermaphroditism, and scrotal anomalies. We report a case of 6 years old male child with right undescended testis and empty left scrotum. Diagnosis was confirmed preoperatively by ultrasound followed by open inguinal exploration for orchidopexy.
\end{abstract}

KEYWORDS - Right undescended testis, Empty left scrotum, Orchidopexy

\section{INTRODUCTION}

Transverse Testicular Ectopia (TTE) is a rare congenital anomaly and is also referred to as testicular pseudoduplication, unilateral double testis, and transverse aberrant testicular with mal-descent. Associated abnormalities may include persistent mullerian duct syndrome, true hermaphroditism, inguinal hernia, hypospadias, pseudohermaphroditism and scrotal anomalies $(1,2)$.

Transverse Testicular Ectopia is an uncommon anatomical abnormality where both the gonads migrate towards the same hemiscrotum. Approximately 100 cases have been reported in literature (1) and it was first described by Von Lennhosek Embryologically, several theories regarding the origin of Transverse Testicular Ectopia have been suggested including adhesion and fusion of developing Wolffian canals, aberrant gubernaculum, testicular adhesions, defective formation of the internal inguinal ring and traction on a testis by persistent mullerian structures. Persistent mullerian duct structures (PMDS) may result from the failure of synthesis or release of mullerian duct inhibitory factor (MIF), the failure of end organs to respond to Mullerian Inhibiting Factor, or defect in the timing of the release of Mullerian Inhibiting Factor. It seems possible that the mechanical effect of the persistent mullerian duct structures prevents the testicular descent or leads to both testicles descending toward the same hemiscrotum producing Transverse Testicular Etopia (3). The association with cryptorchidism is accompanied by an increase in malignancy potential of crossed ectopic testes. The ectopic testis may lie in opposite hemiscrotum, in the inguinal canal or at the deep inguinal ring. An inguinal hernia is invariably present on the side to which the ectopic testis is migrated. On the basis of the presence of various associatedanomalies, TTE has been classified into 3 types (4):

Type 1 - Accompanied only by hernia (40\% to $50 \%)$ (C) International Journal of Recent of Surgical and Medical Science | Jul-Dec 2015 | Vol 1 | Issue 1 | (C) Society for Medicine \& Surgical Update (SMSU) www.ijrsms.com 
Type 2- Accompanied by persistent or rudimentary mullerian duct structures $(30 \%)$

Type 3- Associated with disorders other than persistent mullerian remnants (hypospadias, pseudohermaphroditism, and scrotal abnormalities) $(20 \%)$.

\section{CASE PRESENTATION}

A 6-year-old male child presented with right undescended testis. On local examination right testis was found in right inguinal region and was palpable. Left scrotal sac was empty. On ultrasound examination, two testis were found in right inguinal region, one above the other. On inguinal exploration the two testis were found in right inguinal region. Both the testis and the associated hernial sac was dissected and transfixed. Both testis were pulled down, one testis was fixed in right dartos pouch, the other testis was fixed obliquely in the left dartos pouch by trans septal approach.

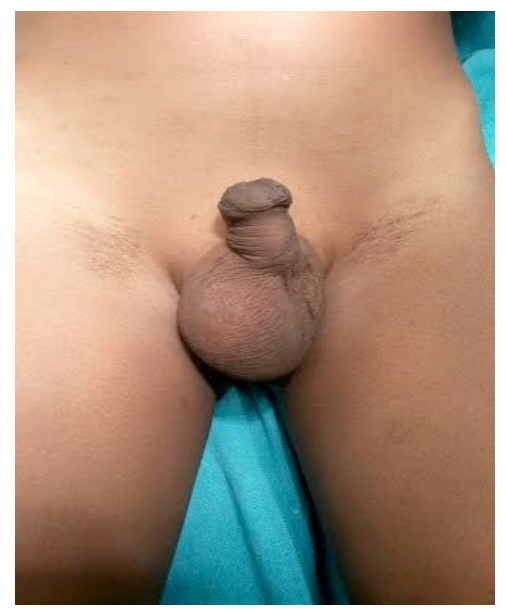

Figure 1 - Showing the Pre - operative photograph

\section{DISCUSSION}

The mean age at presentation of Crossed Testicular Ectopia is 4 years and the clinical presentation generally includes an inguinal hernia on one side and a contralateral or sometimes a bilateral cryptorchidism $(5,6)$.

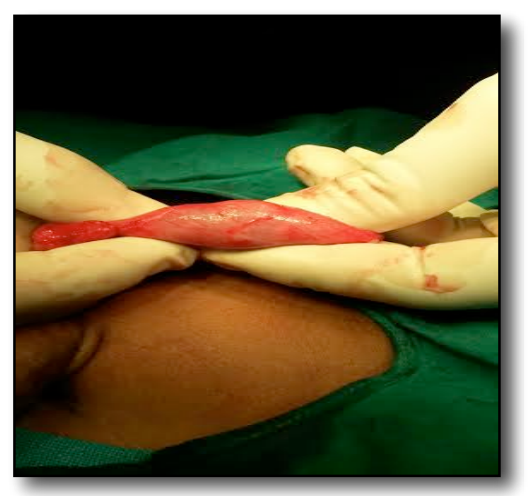

Figure 2 - The pouch containing two testis

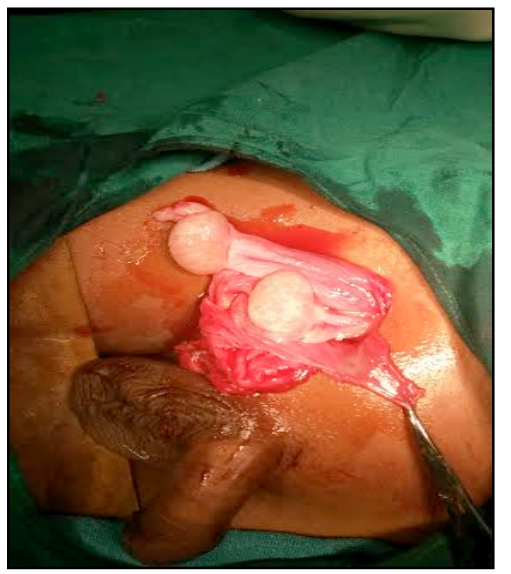

Figure 3 - Testis along with the hernia sac

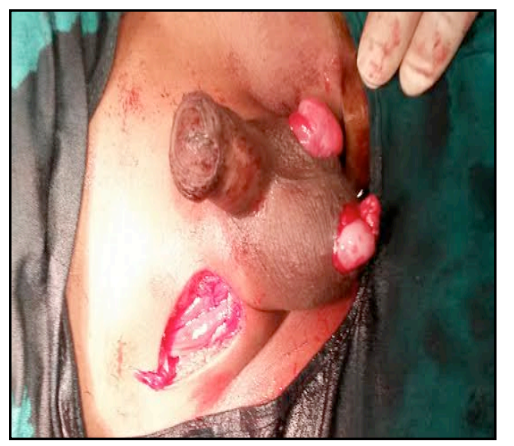

Figure 4 - After trans fixation of testis in the scrotum

Usually, the correct diagnosis cannot be made before surgical exploration. The diagnosis of Transverse Testicular Ectopia can be made preoperatively by close clinical examination and use of ultrasonography by an experienced sonographer (7). Patients with Transverse Testicular Ectopia are at an increased risk of 
malignant transformation with the overall incidence of malignant transformation of gonads approximately $18 \%$ (8). There have also been reports of associated embryonal carcinoma (9) seminoma, yolk sac tumor (10), and teratoma (8).

Walsh et al. (11) concluded that testicular cancer was nearly 6 times more likely to develop in cryptorchid cases where operations were delayed until after 10 to 11 years of age. Wood et al. (12) showed that risk of malignancy in undescended testicles decreased if orchidopexy performed before ages 10 to 12 years. In patients with Transverse Testicular Ectopia, disorders of urinary tract system have also been reported (13).

Once diagnosis of Transverse Testicular Ectopia is made, orchidopexy is recommended for the preservation of fertility Laparoscopy is useful for both diagnosis and its treatment and associated anomalies (14). Management for testicular ectopia is either trans-septal or extra-peritoneal transposition orchidopexy $(15,16)$ a search for mullerian remnants or other anomalies, and a long-term postoperative follow-up. In the extra-peritoneal technique the testis is brought to the contralateral hemiscrotum crossing the root of penis. In the trans-septal technique the testis should traverse the scrotal mediastinum (septum) to be fixed in it. Transverse Testicular Ectopia may also be misdiagnosed as an inguinal hernia, intersex (14) or present as an irreducible hernia requiring urgent surgery (17). Transverse Testicular Ectopia associated with fused vas deferens is extremely rare. This condition may hinder the testis from being placed into the scrotum during orchiopexy (18)
The present case is a rare case in the literature ,only 100 cases have been reported till date. Surgeons who frequently repair inguinal hernias should be aware of the appropriate surgical management options available to them when this condition is unexpectedly identified during inguinal exploration. It is thought that it will be beneficial to keep this rare clinical entity in mind in cases of bilateral undescended testis.

\section{REFRENCES}

1. Fourcroy JL, Belman AB (1982) Transverse testicular ectopia with persistent Mullerian duct. Urology 19(5): 536-538.

2. Von Lenhossek MN (1886) Ectopia testis transversa. Anat Anz 1: 376- 381.

3. Karnak I, Tanyel FC, Akcoren Z, Hicsonmez A (1997) Transverse testicular ectopia with persistent mullerian duct syndrome. J Pediatr Surg 32(9): $1362-$ 1364

4. Gauderer MW, Grisoni ER, Stellato TA, Ponsky JL, Izant RJ Jr (1982) Transverse testicular Ectopia. J Pediatr Surg 17(1): 43-47.

5. Kerigh FB, Rezaei MM (2005) Crossed testicular ectopia: a case report. Urol J 2(4):222-223.

6. Acikalin MF, Pasaoglu O, Tokar B, Ilgici D, Ilhan H (2004) Persistent Mullerian duct syndrome with transverse testicular ectopia: a case report with literature review. Turk J Med Sci 34:333-336.

7. Nam YS, Baik HK, Kim SJ, Lee HK, Park HK (1998) Transverse testicular ectopia found by preoperative ultrasonography. J Korean Med Sci 13(3): 328-330.

8. Berkmen F (1997) Persistent mullerian duct syndrome with or without transverse testicular ectopia and testis tumours. Br J Urol 79(1): 122-126.

9. Melman A, Leiter E, Perez JM, Driscoll D, Palmer C (1981) The influence of neonatal orchiopexy upon the testis in persistent Mullerian duct syndrome. J Urol 125(6): 856-858.

10. Eastham JA, McEvoy K, Sullivan R, Chandrasoma $P$ (1992) A case of simultaneous bilateral nonseminomatous testicular tumors in persistent mullerian duct syndrome. J Urol 148(2 Pt 1): 407-408.

11. Walsh TJ, Dall'Era MA, Croughan MS, Carroll PR, Turek PJ (2007) Prepubertal orchiopexy for 
cryptorchidism may be associated with lower risk of testicular cancer. J Urol 178(4 Pt 1): 1440-1446.

12. Wood HM, Elder JS (2009) Cryptorchidism and testicular cancer: separating fact from fiction. J Urol 181(2): 452-461.

13. Tolete-Velcek F, Bernstein MO, Hansbrough F (1988) Crossed testicular ectopia with bilateral duplication of the vasa deferentia: an unusual finding in cryptorchism. J Pediatr Surg 23(7): 641-643.

14. Gornall PG, Pender DJ (1987) Crossed testicular ectopia detected by laparoscopy. Br J Urol 59(3): 283.
15. Esteves E, Pinus J, Maranhao RF, Abib Sde C, Pinus J (1995) Crossed testicular ectopia. Sao Paulo Med J 113(4): 935-940

16. Pandey A, Gupta DK, Gangopadhyay AN, Sharma SP (2009) Misdiagnosed transverse testicular ectopia: a rare entity. Hernia 13(3): 305-307

17. Vaos G, Zavras N (2004) Irreducible inguinal hernia due to crossed testicular ectopia in an infant. Hernia 8(4): 397-398.

18. Chacko JK, Furness PD 3rd, Mingin GC (2006) Presentation of fused vas deferens. Urology 67(5): 1085.e17-1085.e18.

How to cite this article - Priya N, Luthra L, Agrawal S, Crossed Testicular Ectopia - A Rare Anamoly, IJRSMS, 2015;01(1): 15 - 18 